\title{
ON A FIBONACCI-LIKE SEQUENCE ASSOCIATED WITH $K$-LUCAS SEQUENCE
}

\author{
A. A. Wani, V. H. Badshah, S. Halici, P. Catarino
}

ABstract. In the present article we consider a new generalization of classical Fibonacci sequence and we call it as Fibonacci-Like sequence $\left\langle V_{k, n}\right\rangle$ and then we study Fibonacci-Like sequence $\left\langle V_{k, n}\right\rangle$ and $k$-Lucas sequence $\left\langle L_{k, n}\right\rangle$ side by side by introducing two special matrices for these two sequences. After that by using these matrices we obtain Binet formulae for Fibonacci-Like sequence and for $k$-Lucas sequence, we also give Cassini's identity for Fibonacci-Like sequence.

2010 Mathematics Subject Classification: 11B37, 11B39.

Keywords: Fibonacci Sequence, Generalized Fibonacci Sequence and $k$-Lucas Sequence.

\section{INTRODUCTION}

The Fibonacci sequence $\left\langle F_{n}\right\rangle_{n \geq 0}$ is the sequence of integers given by

$$
F_{n+2}=F_{n+1}+F_{n}, F_{0}=0 \text { and } F_{1}=1
$$

Classical Fibonacci numbers have been generalized by lot of the authors in different ways. The two most important generalizations of Fibonacci numbers are $k$-Fibonacci numbers $\left\langle F_{k, n}\right\rangle[1]$ and $k$-Lucas numbers $\left\langle L_{k, n}\right\rangle[2]$.

Especially some authors used matrix technique to study these numbers. In 1979, Silvester [3] obtained some properties of classical Fibonacci numbers by matrix methods, particularly here the author used diagonaliztion of $2 \times 2$ matrix to obtain Binet formula for classical Fibonacci numbers. In [4] Kilic introduced Binet formula, sums, combinatorial representations and generating function for the generalized Fibonacci $p$-numbers by using matrix technique, these numbers are defined by the following recurrence relation:

$$
F_{p}(n)=F_{p}(n-1)+F_{p}(n-p-1), n>p+1
$$


With initial conditions

$$
F_{p}(1)=F_{p}(2)=\cdots=F_{p}(p)=F_{p}(p+1)=1
$$

Jun and Choi [5] presented some basic properties for generalized Fibonacci sequence $\left\langle q_{n}\right\rangle_{n \in \mathbb{N}}$. Ying et al. [6] studied the generalized Fibonacci numbers by two different matrix methods via the method of diagonalization and the method of matrix collation. Akyuz and Halici [7] considered Horadam sequence (see [7] ) and showed interest towards two cases of Horadam sequence i.e. the sequences $\left\langle U_{n}\right\rangle$ and $\left\langle V_{n}\right\rangle$, which are delineated by

$$
\begin{aligned}
& U_{n}=p U_{n-1}-q U_{n-2}, n \geq 2 \text { and } U_{0}=0, U_{1}=1 \\
& V_{n}=p V_{n-1}-q V_{n-2}, n \geq 2 \text { and } V_{0}=2, V_{1}=p
\end{aligned}
$$

where $p$ and $q$ are integers with $p>0, q \neq 0$, in [7] the authors derived some combinatorial identities, the determinant and the $n^{\text {th }}$ power of $2 \times 2$ matrix. In [8] the authors found some well-known equalities and Binet formula for Jacobsthal numbers by matrix methods. Some divisible properties have been obtained for the generalized Fibonacci sequence by Yalciner [9]. Borges et al. [10] obtained Cassini's identities and Binet formulae for $k$-Fibonacci and $k$-Lucas sequences by using some tools of matrix algebra. In $[11,12]$ the authors also used matrix terminology to deduce Cassini's identities and Binet formulae for the $k$-Pell and $k$-Pell-Lucas Sequences. Srisawat and Sripad [13] applied matrix technique to investigate some generalizations of Pell and Pell-Lucas numbers as $(s, t)$-Pell and $(s, t)$-Pell-Lucas numbers, these numbers are defined respectively by

$$
\begin{aligned}
& \mathcal{P}_{n}(s, t)=2 s \mathcal{P}_{n-1}(s, t)+t \mathcal{P}_{n-2}(s, t) \text { for } n \geq 2 \\
& \mathcal{Q}_{n}(s, t)=2 s \mathcal{Q}_{n-1}(s, t)+t \mathcal{Q}_{n-2}(s, t) \text { for } n \geq 2
\end{aligned}
$$

with initial conditions $\mathcal{P}_{0}(s, t)=0, \mathcal{P}_{1}(s, t)=1$ and $\mathcal{Q}_{0}(s, t)=2, \mathcal{P}_{1}(s, t)=2 s$.

\section{Preliminaries}

First of all, we consider a sequence $\left\langle G_{k, n}\right\rangle$ which is defined by the following recurrence relation:

$$
G_{k, n+2}=k G_{k, n+1}+G_{k, n}, n \geq 1 \text { and } G_{k, 0}=a, G_{k, 1}=b
$$

where $k \in \mathbb{R}^{+}$and $a, b \in \mathbb{Z}^{+}$.

For the present study we are interested in the two cases of the sequence $\left\langle G_{k, n}\right\rangle$ : 
A. A. Wani, V. H. Badshah, S. Halici, P. Catarino - On A Fibonacci-Like ...

(i) Fibonacci-Like sequence $\left\langle V_{k, n}\right\rangle$ is defined by the following equation:

$$
V_{k, n+2}=k V_{k, n+1}+V_{k, n}, n \geq 0 \text { and } V_{k, 0}=2 m, V_{k, 1}=p+m k
$$

(ii) $k$-Lucas sequence $\left\langle L_{k, n}\right\rangle[2]$ is defined recurrently by

$$
L_{k, n+2}=k L_{k, n+1}+L_{k, n}, n \geq 0 \text { and } L_{k, 0}=2, L_{k, 1}=k
$$

Both the recurrence relations (2.2) and (2.3) have same characteristic equation $x^{2}-$ $k x-1=0$. Let $r$ and $s$ be its roots and are given as

$$
r=\frac{k+\sqrt{k^{2}+4}}{2} \text { and } s=\frac{k-\sqrt{k^{2}+4}}{2}
$$

We can see easily $r$ and $s$ holds the following properties:

(a) $r s=-1, r+s=k$ and $r-s=\sqrt{k^{2}+4}$

(b) $r^{2}-1=k r$ and $s^{2}-1=k s$

(c) $r^{2}+1=k r+2=(r-s) r$ and $s^{2}+1=k s+2=-(r-s) s$

Also we introduce two special $2 \times 2$ matrices $V$ and $L$ which are given by

$$
V=\left[\begin{array}{ll}
k & 1 \\
1 & 0
\end{array}\right] \text { and } L=\left[\begin{array}{cc}
k^{2}+2 & k \\
k & 2
\end{array}\right]
$$

\section{3. $n^{\text {th }}$ Powers of The Matrices}

Theorem 1. For $n \in \mathbb{Z}^{+}$, we have the following result

$$
V^{n}=\left[\begin{array}{cc}
\frac{2 m V_{k, n+2}-(p+m k) V_{k, n+1}}{m^{2} k^{2}+4 m^{2}-p^{2}} & \frac{2 m V_{k, n+1}-(p+m k) V_{k, n}}{m^{2} k^{2}+4 m^{2}-p^{2}} \\
\frac{2 m V_{k, n+1}-(p+m k) V_{k, n}}{m^{2} k^{2}+4 m^{2}-p^{2}} & \frac{2 m V_{k, n}-(p+m k) V_{k, n-1}}{m^{2} k^{2}+4 m^{2}-p^{2}}
\end{array}\right]
$$

Proof. We use principle of mathematical induction on $n$. Certainly the result is true for $n=1$. 
Assume that the result is true for all values $j$ less than or equal $n$ and then

$$
\begin{aligned}
V^{n+1} & =\left[\begin{array}{ll}
\frac{2 m V_{k, n+2}-(p+m k) V_{k, n+1}}{m^{2} k^{2}+4 m^{2}-p^{2}} & \frac{2 m V_{k, n+1}-(p+m k) V_{k, n}}{m^{2} k^{2}+4 m^{2}-p^{2}} \\
\frac{2 m V_{k, n+1}-(p+m k) V_{k, n}}{m^{2} k^{2}+4 m^{2}-p^{2}} & \frac{2 m V_{k, n}-(p+m k) V_{k, n-1}}{m^{2} k^{2}+4 m^{2}-p^{2}}
\end{array}\right]\left[\begin{array}{ll}
k & 1 \\
1 & 0
\end{array}\right] \\
V^{n+1} & =\left(m^{2} k^{2}+4 m^{2}-p^{2}\right)\left[\begin{array}{ll}
a_{1} & a_{2} \\
a_{3} & a_{4}
\end{array}\right]\left[\begin{array}{ll}
k & 1 \\
1 & 0
\end{array}\right] \\
V^{n+1} & =\left(m^{2} k^{2}+4 m^{2}-p^{2}\right)\left[\begin{array}{ll}
k a_{1}+a_{2} & a_{1} \\
k a_{3}+a_{4} & a_{3}
\end{array}\right]
\end{aligned}
$$

Here

$$
\begin{aligned}
k a_{1}+a_{2} & =2 m\left(k V_{k, n+2}+V_{k, n+1}\right)-(p+m k)\left(k V_{k, n+1}+V_{k, n}\right) \\
& =2 m V_{k, n+3}-(p+m k) V_{k, n+2}
\end{aligned}
$$

and

$$
\begin{aligned}
k a_{3}+a_{4} & =2 m\left(k V_{k, n+1}+V_{k, n}\right)-(p+m k)\left(k V_{k, n}+V_{k, n-1}\right) \\
& =2 m V_{k, n+2}-(p+m k) V_{k, n+1}
\end{aligned}
$$

Hence

$$
V^{n+1}=\left[\begin{array}{ll}
\frac{2 m V_{k, n+3}-(p+m k) V_{k, n+2}}{m^{2} k^{2}+4 m^{2}-p^{2}} & \frac{2 m V_{k, n+2}-(p+m k) V_{k, n+1}}{m^{2} k^{2}+4 m^{2}-p^{2}} \\
\frac{2 m V_{k, n+2}-(p+m k) V_{k, n+1}}{m^{2} k^{2}+4 m^{2}-p^{2}} & \frac{2 m V_{k, n+1}-(p+m k) V_{k, n}}{m^{2} k^{2}+4 m^{2}-p^{2}}
\end{array}\right]
$$

as required.

Corollary 2. For $\lim _{n \rightarrow \infty} \frac{V_{k, n+1}}{V_{k, n}}=r$, we have

$$
r^{2}-k r-1=0
$$

Proof. To prove the required result, we should use the concept of limits. Since

$$
\lim _{n \rightarrow \infty} \frac{V^{n}}{V_{k, n-1}}=\lim _{n \rightarrow \infty}\left[\begin{array}{ll}
\frac{2 m V_{k, n+2}-(p+m k) V_{k, n+1}}{\left(m^{2} k^{2}+4 m^{2}-p^{2}\right) V_{k, n-1}} & \frac{2 m V_{k, n+1}-(p+m k) V_{k, n}}{\left(m^{2} k^{2}+4 m^{2}-p^{2}\right) V_{k, n-1}} \\
\frac{2 m V_{k, n+1}-(p+m k) V_{k, n}}{\left(m^{2} k^{2}+4 m^{2}-p^{2}\right) V_{k, n-1}} & \frac{2 m V_{k, n}-(p+m k) V_{k, n-1}}{\left(m^{2} k^{2}+4 m^{2}-p^{2}\right) V_{k, n-1}}
\end{array}\right]
$$


Now

$$
\begin{aligned}
\lim _{n \rightarrow \infty} \frac{2 m V_{k, n+2}-(p+m k) V_{k, n+1}}{V_{k, n-1}} & =r^{2}(2 m r-p-m k) \\
\lim _{n \rightarrow \infty} \frac{2 m V_{k, n+1}-(p+m k) V_{k, n}}{V_{k, n-1}} & =r(2 m r-p-m k) \\
\lim _{n \rightarrow \infty} \frac{2 m V_{k, n}-(p+m k) V_{k, n-1}}{V_{k, n-1}} & =2 m r-p-m k
\end{aligned}
$$

Therefore,

$$
\begin{aligned}
& \lim _{n \rightarrow \infty} \frac{V^{n}}{V_{k, n-1}}=\left(m^{2} k^{2}+4 m^{2}-p^{2}\right)^{-1}\left[\begin{array}{cc}
r^{2}(2 m r-p-m k) & r(2 m r-p-m k) \\
r(2 m r-p-m k) & 2 m r-p-m k
\end{array}\right] \\
& \left(m^{2} k^{2}+4 m^{2}-p^{2}\right)^{-1}\left[\begin{array}{cc}
r^{2}(2 m r-p-m k) & r(2 m r-p-m k) \\
r(2 m r-p-m k) & 2 m r-p-m k
\end{array}\right] \\
& =\left(m^{2} k^{2}+4 m^{2}-p^{2}\right)^{-1}\left[\begin{array}{cc}
(k r+1)(2 m r-p-m k) & r(2 m r-p-m k) \\
r(2 m r-p-m k) & 2 m r-p-m k
\end{array}\right]
\end{aligned}
$$

If we equate determinant on both sides, we obtain

$$
\begin{aligned}
& 0=k r+1-r^{2} \\
& r^{2}-k r-1=0
\end{aligned}
$$

Hence the result.

Theorem 3. For $n \in \mathbb{N}$, the following result holds

$$
L^{n}= \begin{cases}(r-s)^{n-1} U^{n} & \text { if } n \text { is odd } \\ (r-s)^{n} V^{n} & \text { if } n \text { is even }\end{cases}
$$

where $U^{n}=\left[\begin{array}{cc}L_{k, n+1} & L_{k, n} \\ L_{k, n} & L_{k, n-1}\end{array}\right]$

Proof. To prove the result, we use induction on $n$. First, we consider odd $n$. Let $n=1$, we have

$$
L=\left[\begin{array}{cc}
k^{2}+2 & k \\
k & 2
\end{array}\right]=\left[\begin{array}{cc}
L_{k, 2} & L_{k, 1} \\
L_{k, 1} & L_{k, 0}
\end{array}\right]
$$


Let us suppose that the result is true for all odd values $i$ less than or equal $n$ and then

$$
\begin{aligned}
L^{n+2} & =(r-s)^{\frac{n-1}{2}} U^{n} L^{2} \\
& =(r-s)^{\frac{n+1}{2}} U^{n} V^{2} \\
& =(r-s)^{\frac{n+1}{2}} U^{n+2}
\end{aligned}
$$

as required.

Now we consider even $n$. Let $n=2$, we have

$$
\begin{aligned}
L^{2} & =\left[\begin{array}{cc}
k^{4}+5 k^{2}+4 & k^{3}+4 k \\
k^{3}+4 k & k^{2}+4
\end{array}\right] \\
& =(r-s)\left[\begin{array}{cc}
k^{2}+1 & k \\
k & 1
\end{array}\right] \\
& =(r-s) V^{2}
\end{aligned}
$$

Assume that the result is true for all even values $j$ less than or equal $n$ and then

$$
\begin{aligned}
L^{n+2} & =(r-s)^{\frac{n}{2}} V^{n} L^{2} \\
& =(r-s)^{\frac{n+2}{2}} V^{n} V^{2} \\
& =(r-s)^{\frac{n+2}{2}} V^{n+2}
\end{aligned}
$$

as needed.

Lemma 4. For $n \geq 0$, we have

$$
V^{n}\left[\begin{array}{c}
V_{k, 1} \\
V_{k, 0}
\end{array}\right]=\left[\begin{array}{c}
V_{k, n+1} \\
V_{k, n}
\end{array}\right]
$$

Proof. Here we shall use induction on $n$. Indeed the result is true for $n=0$. Suppose the result is true for all values $i$ less than or equal $n$ and then

$$
\begin{aligned}
V^{n+1}\left[\begin{array}{l}
V_{k, 1} \\
V_{k, 0}
\end{array}\right] & =V V^{n}\left[\begin{array}{l}
V_{k, 1} \\
V_{k, 0}
\end{array}\right] \\
& =\left[\begin{array}{ll}
k & 1 \\
1 & 0
\end{array}\right]\left[\begin{array}{c}
V_{k, n+1} \\
V_{k, n}
\end{array}\right] \\
& =\left[\begin{array}{c}
k V_{k, n+1}+V_{k, n} \\
V_{k, n+1}
\end{array}\right]
\end{aligned}
$$




$$
=\left[\begin{array}{l}
V_{k, n+2} \\
V_{k, n+1}
\end{array}\right]
$$

as required.

\section{Binet Formulae}

In this section we present Binet formulae for $k$-Lucas sequence $\left\langle L_{k, n}\right\rangle$ and for FibonacciLike sequence $\left\langle V_{k, n}\right\rangle$. The most worth noticing point is here that we obtain Binet formula for $k$-Lucas in a different way that did the authors in $[2,10]$.

Theorem 5. For $n \in \mathbb{Z}_{0}$, the $n^{\text {th }}$ terms for $\left\langle L_{k, n}\right\rangle$ and $\left\langle V_{k, n}\right\rangle$ are respectively given by

$$
\begin{aligned}
& L_{k, n}=r^{n}+s^{n} \\
& V_{k, n}=p \frac{r^{n}-s^{n}}{r-s}+m\left(r^{n}+s^{n}\right)
\end{aligned}
$$

where $r$ and $s$ are determined from equation (2.4).

Proof. To prove the needed result, we diagonalize the matrix $L$. Sine $L$ is a square matrix, by the Cayley Hamilton theorem the characteristic equation of $L$ is given by

$$
\begin{aligned}
\operatorname{det}\left(L-x I_{2}\right) & =0 \\
{\left[\begin{array}{cc}
k^{2}+2-x & k \\
k & 2-x
\end{array}\right] } & =0 \\
x^{2}-\left(k^{2}+4\right) x+\left(k^{2}+4\right) & =0 \\
x^{2}-(r-s) x+(r-s) & =0
\end{aligned}
$$

This is the characteristic equation of $L$. Let $u$ and $v$ be the eigen values of equation (4.3) and are given by

$$
\begin{aligned}
u & =\frac{k(r-s)+(r-s)^{2}}{2} \\
& =(r-s)\left[\frac{k+(r-s)}{2}\right] \\
& =r(r-s)
\end{aligned}
$$


and

$$
\begin{aligned}
v & =\frac{-k(r-s)+(r-s)^{2}}{2} \\
& =-(r-s)\left[\frac{k-(r-s)}{2}\right] \\
& =-s(r-s)
\end{aligned}
$$

Now the eigen vector corresponding to eigen value $u$ is given by the following equation:

$$
\left(L-u I_{2}\right) A
$$

where $A$ is the column vector of order $2 \times 1$. Then

$$
\begin{aligned}
& {\left[\begin{array}{cc}
k^{2}+2-u & k \\
k & 2-u
\end{array}\right]\left[\begin{array}{l}
A_{1} \\
A_{2}
\end{array}\right]=0} \\
& {\left[\begin{array}{c}
\left(k^{2}+4-u\right) A_{1}+k A_{2} \\
k A_{1}+(2-u) A_{2}
\end{array}\right]=0}
\end{aligned}
$$

Consider the system

$$
\begin{aligned}
\left(k^{2}+4-u\right) A_{1}+k A_{2} & =0 \\
k A_{1}+(2-u) A_{2} & =0
\end{aligned}
$$

We assume that $A_{2}=l$ in equation (4.4), we achieve

$$
A_{1}=\frac{(u-2) l}{k}=\frac{\left(r^{2}-1\right) l}{k}=l r
$$

Thus the eigen vectors corresponding to $u$ are of kind $\left[\begin{array}{l}l r \\ l\end{array}\right]$. For particular $l=1$, the eigen vector assigning to $u$ is $\left[\begin{array}{l}r \\ 1\end{array}\right]$. Similarly the eigen vector assigning to $v$ is $\left[\begin{array}{l}s \\ 1\end{array}\right]$. Let $P$ be matrix of eigen vectors, $P=\left[\begin{array}{ll}r & s \\ 1 & 1\end{array}\right]$ and $P^{-1}=(r-s)^{-1}\left[\begin{array}{cc}1 & -s \\ -1 & -r\end{array}\right]$. Now we consider the diagonal matrix $D$, in which eigen values of $L$ are on the main diagonal, $D=\left[\begin{array}{cc}r(r-s) & 0 \\ 0 & -s(r-s)\end{array}\right]$. Then by the principle of matrix diagonalization $[14,15]$, we have

$$
L=P D P^{-1}
$$




$$
\begin{aligned}
L^{n} & =P D^{n} P^{-1} \\
& =(r-s)^{-1}\left[\begin{array}{ll}
r & s \\
1 & 1
\end{array}\right]\left[\begin{array}{cc}
r^{n}(r-s)^{n} & 0 \\
0 & (-1)^{n} s^{n}(r-s)^{n}
\end{array}\right]\left[\begin{array}{cc}
1 & -s \\
-1 & r
\end{array}\right] \\
& =(r-s)^{n-1}\left[\begin{array}{cc}
r^{n+1} & (-1)^{n} s^{n+1} \\
r^{n} & (-1)^{n} s^{n}
\end{array}\right]\left[\begin{array}{cc}
1 & -s \\
-1 & r
\end{array}\right] \\
& =(r-s)^{n-1}\left[\begin{array}{cc}
r^{n+1}-(-1)^{n} s^{n+1} & -s r^{n+1}+(-1)^{n} r s^{n+1} \\
r^{n}-(-1)^{n} s^{n} & -s r^{n}+(-1)^{n} r s^{n}
\end{array}\right] \\
& =(r-s)^{n-1}\left[\begin{array}{cc}
r^{n+1}-(-1)^{n} s^{n+1} & r^{n}-(-1)^{n} s^{n} \\
r^{n}-(-1)^{n} s^{n} & r^{n-1}-(-1)^{n} s^{n-1}
\end{array}\right]
\end{aligned}
$$

If $n$ is odd then by equation (3.3), we get

$$
U^{n}=\left[\begin{array}{cc}
r^{n+1}+s^{n+1} & r^{n}+s^{n} \\
r^{n}+s^{n} & r^{n-1}+s^{n-1}
\end{array}\right]
$$

By equating corresponding terms of the matrices, we have

$$
L_{k, n}=r^{n}+s^{n}
$$

This is the required Binet's formula for $k$-Lucas sequence.

if $n$ is even then again by equation (3.3), we achieve

$$
V^{n}=(r-s)^{-1}\left[\begin{array}{cc}
r^{n+1}-s^{n+1} & r^{n}-s^{n} \\
r^{n}-s^{n} & r^{n-1}-s^{n-1}
\end{array}\right]
$$

By using lemma (4), we obtain

$$
\begin{aligned}
{\left[\begin{array}{c}
V_{k, n+1} \\
V_{k, n}
\end{array}\right] } & =(r-s)^{-1}\left[\begin{array}{cc}
r^{n+1}-s^{n+1} & r^{n}-s^{n} \\
r^{n}-s^{n} & r^{n-1}-s^{n-1}
\end{array}\right]\left[\begin{array}{c}
p+m k \\
2 m
\end{array}\right] \\
& =(r-s)^{-1}\left[\begin{array}{cc}
b_{1} & b_{2} \\
r^{n}-s^{n} & r^{n-1}-s^{n-1}
\end{array}\right]\left[\begin{array}{c}
p+m k \\
2 m
\end{array}\right]
\end{aligned}
$$

where $b_{1}$ and $b_{2}$ are the corresponding terms of the matrix. Thus

$$
\left[\begin{array}{c}
V_{k, n+1} \\
V_{k, n}
\end{array}\right]=(r-s)^{-1}\left[\begin{array}{c}
(p+m k) b_{1}+2 m b_{2} \\
(p+m k)\left(r^{n}-s^{n}\right)+2 m\left(r^{n-1}-s^{n-1}\right)
\end{array}\right]
$$


A. A. Wani, V. H. Badshah, S. Halici, P. Catarino - On A Fibonacci-Like ...

$$
\begin{aligned}
& =(r-s)^{-1}\left[\begin{array}{c}
(p+m k) b_{1}+2 m b_{2} \\
p\left(r^{n}-s^{n}\right)+m k r^{n}+2 m r^{n-1}-m k s^{n}-2 m s^{n-1}
\end{array}\right] \\
& =(r-s)^{-1}\left[\begin{array}{c}
(p+m k) b_{1}+2 m b_{2} \\
p\left(r^{n}-s^{n}\right)+m r^{n-1}(k r+2)-m s^{n-1}(k s+2)
\end{array}\right] \\
& =(r-s)^{-1}\left[\begin{array}{c}
(p+m k) b_{1}+2 m b_{2} \\
p\left(r^{n}-s^{n}\right)+m(r-s) r^{n}+m(r-s) s^{n}
\end{array}\right]
\end{aligned}
$$

Equating corresponding terms on both sides, we get

$$
V_{k, n}=p \frac{r^{n}-s^{n}}{r-s}+m\left(r^{n}+s^{n}\right)
$$

This is the Binet's formula for Fibonacci-Like sequence.

Now we present a result which establishes a relation between Fibonacci-Like sequence $\left\langle V_{k, n}\right\rangle$ and $k$-Lucas sequence.

Corollary 6. For $n \in \mathbb{Z}^{+}$, the following result holds

$$
\begin{aligned}
& 2 m V_{k, n+2}-(p+m k) V_{k, n+1}+2 m V_{k, n}-(p+m k) V_{k, n-1} \\
& =\left(m^{2} k^{2}+4 m^{2}-p^{2}\right) L_{k, n}
\end{aligned}
$$

Proof. If we equate corresponding terms of matrices in the equation (4.5), we get

$$
\frac{2 m V_{k, n+2}-(p+m k) V_{k, n+1}}{p^{2} m^{2}+4 m^{2}-p^{2}}=\frac{r^{n+1}-s^{n+1}}{r-s}
$$

and

$$
\frac{2 m V_{k, n}-(p+m k) V_{k, n-1}}{p^{2} m^{2}+4 m^{2}-p^{2}}=\frac{r^{n-1}-s^{n-1}}{r-s}
$$

Therefore,

$$
\begin{aligned}
& \frac{2 m V_{k, n+2}-(p+m k) V_{k, n+1}+2 m V_{k, n}-(p+m k) V_{k, n-1}}{p^{2} m^{2}+4 m^{2}-p^{2}} \\
& =\frac{r^{n-1}\left(r^{2}+1\right)-s^{n-1}\left(s^{2}+1\right)}{r-s} \\
& =\frac{r^{n}(r-s)+s^{n}(r-s)}{r-s}
\end{aligned}
$$

Thus

$$
\begin{aligned}
& 2 m V_{k, n+2}-(p+m k) V_{k, n+1}+2 m V_{k, n}-(p+m k) V_{k, n-1} \\
& =\left(m^{2} k^{2}+4 m^{2}-p^{2}\right) L_{k, n}
\end{aligned}
$$

Hence the result. 


\section{Cassini's Identity for $\left\langle V_{k, n}\right\rangle$}

In this section we obtain Cassinin's identity for Fibonacci-Like sequence by using matrix $V$.

Theorem 7. For $n \geq 1$, we have

$$
V_{k, n}^{2}-V_{k, n+1} V_{k, n-1}=(-1)^{n}\left(m^{2} k^{2}+4 m^{2}-p^{2}\right)
$$

Proof.

$$
\begin{aligned}
\operatorname{det}\left(V^{n}\right)= & \left|\begin{array}{ll}
\frac{2 m V_{k, n+2}-(p+m k) V_{k, n+1}}{m^{2} k^{2}+4 m^{2}-p^{2}} & \frac{2 m V_{k, n+1}-(p+m k) V_{k, n}}{m^{2} k^{2}+4 m^{2}-p^{2}} \\
\frac{2 m V_{k, n+1}-(p+m k) V_{k, n}}{m^{2} k^{2}+4 m^{2}-p^{2}} & \frac{2 m V_{k, n}-(p+m k) V_{k, n-1}}{m^{2} k^{2}+4 m^{2}-p^{2}}
\end{array}\right| \\
\operatorname{det}\left(V^{n}\right)= & \left.m^{2} k^{2}+4 m^{2}-p^{2}\right)^{-2}\left\{2 m V_{k, n+2}\left[2 m V_{k, n}-(p+m k) V_{k, n-1}\right]\right. \\
& -(p+m k) V_{k, n+1}\left[2 m V_{k, n}-(p+m k) V_{k, n-1}\right]-\left[2 m V_{k, n+1}\right. \\
& \left.\left.-(p+m k) V_{k, n}\right]^{2}\right\}
\end{aligned}
$$

By using expansion of $V_{k, n+2}$ and $V_{k, n+1}^{2}$, we have

$$
\begin{aligned}
\operatorname{det}\left(V^{n}\right)= & \left.m^{2} k^{2}+4 m^{2}-p^{2}\right)^{-2}\left[4 m^{2} k V_{k, n+1} V_{k, n}-2 m k(p+m k) V_{k, n+1}\right. \\
& V_{k, n-1}+4 m^{2} V_{k, n}^{2}-2 m(p+m k) V_{k, n} V_{k, n-1}+(p+m k)^{2} V_{k, n+1} \\
& V_{k, n-1}-2 m(p+m k) V_{k, n+1} V_{k, n}-4 m^{2} k^{2} V_{k, n}^{2}-4 m^{2} V_{k, n-1}^{2} \\
& \left.-8 m^{2} k V_{k, n} V_{k, n-1}-(p+m k)^{2} V_{k, n}^{2}+4 m(p+m k) V_{k, n+1} V_{k, n}\right] \\
\operatorname{det}\left(V^{n}\right)= & \left.m^{2} k^{2}+4 m^{2}-p^{2}\right)^{-2}\left[4 m^{2} k V_{k, n+1} V_{k, n}-2 m(p+m k) V_{k, n} V_{k, n-1}\right. \\
& +2 m(p+m k) V_{k, n+1} V_{k, n}-4 m^{2} V_{k, n-1}^{2}-8 m^{2} k V_{k, n} V_{k, n-1} \\
- & 2 m k(p+m k) V_{k, n+1} V_{k, n-1}+4 m^{2} V_{k, n}^{2}+(p+m k)^{2} V_{k, n+1} V_{k, n-1} \\
& \left.-4 m^{2} V_{k, n}^{2}-(p+m k)^{2} V_{k, n}^{2}\right] \\
= & \left.m^{2} k^{2}+4 m^{2}-p^{2}\right)^{-2}\left[\left(6 m^{2} k+2 m p\right) V_{k, n+1} V_{k, n}-\left(2 m p+2 m^{2} k\right)\right. \\
& V_{k, n} V_{k, n-1}-4 m^{2} V_{k, n-1}^{2}-8 m^{2} k V_{k, n} V_{k, n-1}-2 m k(p+m k) \\
& V_{k, n+1} V_{k, n-1}+4 m^{2} V_{k, n}^{2}+(p+m k)^{2} V_{k, n+1} V_{k, n-1}-4 m^{2} V_{k, n}^{2} \\
& \left.-(p+m k)^{2} V_{k, n}^{2}\right] \\
= & \left.m^{2} k^{2}+4 m^{2}-p^{2}\right)^{-2}\left(6 m^{2} V_{k, n}^{2}+2 m p k V_{k, n}^{2}-4 m^{2} k V_{k, n+1} V_{k, n-1}\right. \\
& -m^{2} k^{2} V_{k, n+1} V_{k, n-1}+4 m^{2} V_{k, n}^{2}+p^{2} V_{k, n+1} V_{k, n-1}-4 m^{2} k^{2} V_{k, n}^{2}
\end{aligned}
$$




$$
\begin{aligned}
& \left.-p^{2} V_{k, n}^{2}-m^{2} k^{2} V_{k, n}^{2}-2 m p k V_{k, n}^{2}\right) \\
= & \left(m^{2} k^{2}+4 m^{2}-p^{2}\right)^{-2}\left(m^{2} V_{k, n}^{2}+4 m^{2} V^{2} k, n-p^{2} V_{k, n}^{2}-m^{2} k^{2}\right. \\
& \left.V_{k, n+1} V_{k, n-1}-4 m^{2} V_{k, n+1} V_{k, n-1}+p^{2} V_{k, n+1} V_{k, n-1}\right) \\
= & \left(m^{2} k^{2}+4 m^{2}-p^{2}\right)^{-2}\left(m^{2} k^{2}+4 m^{2}-p^{2}\right)\left(V_{k, n}^{2}-V_{k, n+1} V_{k, n-1}\right) \\
= & \left(m^{2} k^{2}+4 m^{2}-p^{2}\right)^{-1}\left(V_{k, n}^{2}-V_{k, n+1} V_{k, n-1}\right)
\end{aligned}
$$

Since $\operatorname{det}\left(V^{n}\right)=(-1)^{n}$, we have

$$
V_{k, n}^{2}-V_{k, n+1} V_{k, n-1}=(-1)^{n}\left(m^{2} k^{2}+4 m^{2}-p^{2}\right)
$$

Hence the result.

From the proof of this theorem, we conclude that

$$
\begin{aligned}
& {\left[2 m V_{k, n+2}-(p+m k) V_{k, n+1}\right]\left[2 m V_{k, n}-(p+m k) V_{k, n-1}\right]} \\
& \quad-\left[2 m V_{k, n+1}-(p+m k) V_{k, n}\right]^{2}=(-1)^{2}\left(m^{2} k^{2}+4 m^{2}-p^{2}\right)
\end{aligned}
$$

\section{Characteristic Equation of $V^{n}$}

In theorem (5) we easily saw the characteristic equation of $V$. But in this section we obtain the characteristic equation for $V^{n}$.

Theorem 8. For $n \in \mathbb{Z}_{0}$, the characteristic equation of $V^{n}$ is given by

$$
x^{2}-L_{k, n} x+(-1)^{n}=0
$$

Proof. Since $V^{n}$ is a square matrix then by Cayley Hamilton theorem, we have

$$
\operatorname{det}\left(V^{n}-x I_{2}\right)=0
$$

Here

$$
\begin{gathered}
\operatorname{det}\left(V^{n}-x I_{2}\right) \\
=\left|\begin{array}{cc}
\frac{2 m V_{k, n+2}-(p+m k) V_{k, n+1}}{m^{2} k^{2}+4 m^{2}-p^{2}}-x & \frac{2 m V_{k, n+1}-(p+m k) V_{k, n}}{m^{2} k^{2}+4 m^{2}-p^{2}} \\
\frac{2 m V_{k, n+1}-(p+m k) V_{k, n}}{m^{2} k^{2}+4 m^{2}-p^{2}} & \frac{2 m V_{k, n}-(p+m k) V_{k, n-1}}{m^{2} k^{2}+4 m^{2}-p^{2}}-x
\end{array}\right| \\
=\left(m^{2} k^{2}+4 m^{2}-p^{2}\right)^{-2}\left\{[ 2 m V _ { k , n + 2 } - ( p + m k ) V _ { k , n + 1 } ] \left[2 m V_{k, n}-(p+m k)\right.\right. \\
\left.V_{k, n-1}\right]-x\left(m^{2} k^{2}+4 m^{2}-p^{2}\right)\left[2 m V_{k, n+2}-(p+m k) V_{k, n+1}\right]-x
\end{gathered}
$$




$$
\begin{aligned}
&\left(m^{2} k^{2}+4 m^{2}-p^{2}\right)\left[2 m V_{k, n}-(p+m k) V_{k, n-1}\right]+x^{2}\left(m^{2} k^{2}+4 m^{2}-p^{2}\right)^{2} \\
&\left.-\left[2 m V_{k, n+1}-(p+m k) V_{k, n}\right]^{2}\right\} \\
&=\left(m^{2} k^{2}+4 m^{2}-p^{2}\right)^{-2}\left\{x^{2}\left(m^{2} k^{2}+4 m^{2}-p^{2}\right)^{2}-x\left(m^{2} k^{2}+4 m^{2}-p^{2}\right)\right. \\
& \quad\left[2 m V_{k, n+2}-(1+m k) V_{k, n+1}+2 m V_{k, n}-(p+m k) V_{k, n-1}\right] \\
& \quad+\left[2 m V_{k, n+2}-(p+m k) V_{k, n+1}\right]\left[2 m V_{k, n}-(p+m k) V_{k, n-1}\right] \\
&\left.\quad-\left[2 m V_{k, n+1}-(p+m k) V_{k, n}\right]^{2}\right\}
\end{aligned}
$$

If we consider corollary (6) and equation (5.2), we get

$$
\begin{aligned}
& \operatorname{det}\left(V^{n}-x I_{2}\right)=\left(m^{2} k^{2}+4 m^{2}-p^{2}\right)^{-2}\left[x^{2}\left(m^{2} k^{2}+4 m^{2}-p^{2}\right)^{2}\right. \\
& \left.\quad-L_{k, n} x\left(m^{2} k^{2}+4 m^{2}-p^{2}\right)^{2}+(-1)^{n}\left(m^{2} k^{2}+4 m^{2}-p^{2}\right)^{2}\right] \\
& =x^{2}-L_{k, n} x+(-1)^{n}
\end{aligned}
$$

Hence the characteristic equation of $V^{n}$ is

$$
x^{2}-L_{k, n} x+(-1)^{n}=0
$$

Hence the result.

\section{REFERENCES}

[1] S. Falcon and A. Plaza, On the Fibonacci $k$-Numbers, Chaos Solitons and Fractals, 32 (2007), 1615-1624.

[2] S. Falcon, On the $k$-Lucas Numbers, Int. J. Contemp. Math. Sciences, 6(21) (2011), 1615-1624.

[3] J. R. Silvester, Fibonacci Numbers by Matrix Methods, The Mathematical Gazette, 63(425) (1979), 181-191.

[4] E. Kilic, The Binet Formula, Sums and Representations of Generalized Fibonacci p-Numbers, Chaos Solitons and Fractals, 32 (2007), 1615-1624.

[5] S. P. Jun and K. H. Choi, Some Properties of the Generalized Fibonacci Sequence $\left\langle q_{n}\right\rangle$ by Matrix Methods, Korean J. Math., 24(4) (2016), 681-691.

[6] S. J. Ying, H. C. Kit , Ibrahim H. and Ahmad N., Algebraic Properties of Generalized Fibonacci Sequence Via Matrix Methods, Journal of Engineering and Applied Sciences, 11(11) (2016), 2396-2401.

[7] Z. Akyuz and S. Halici, Some Identities Deriving from the nth Power of a Special Matrix, Advances in Difference Equations, 2012. 
A. A. Wani, V. H. Badshah, S. Halici, P. Catarino - On A Fibonacci-Like ...

[8] F. Koken and D. Bozkurt, On the Jacobsthal Numbers by Matrix Methods, Int. J. Contemp. Math. Sciences, 3(13) (2008), 605-614.

[9] A. Yalciner, A Matrix Approach for Divisibility Properties of the Generalized Fibonacci Sequence, Discrete Dynamics in Nature and Society, 2013.

[10] A. Borges, P. Catarino, A. P. Aires, P. Vasco, and H. Campos, Two-by-Two Matrices Involving k-Fibonacci and $k$-Lucas Sequences, Applied Mathematical Sciences, 8(34) (2014), 1659-1666.

[11] P. Catarino and P. Vasco, Two-by-Two Matrices Involving k-Fibonacci and $k$ Lucas Sequences, Int. Journal of Math. Analysis, 7(45) (2013), 2209-2215.

[12] P. Catarino and P. Vasco, A Note Involving Two-by-Two Matrices of the $k$ Pell and k-Pell-Lucas Sequences, International Mathematical Forum, 8(32) (2013), 1561-1568.

[13] S. Srisawat and W. Sripad, On the $(s, t)$-Pell and $(s, t)$-Pell-Lucas Numbers by Matrix Methods, Annales Mathematicae et Informaticae, 8(32) (2013), 1561-1568.

[14] http://mathworld.wolfram.com/MatrixDiagonalization.html

[15] http://mathworld.wolfram.com/EigenDecomposition.html

\section{A. A. Wani}

School of Studies in Mathematics, Vikram University Ujjain,

Ujjain, India

email: arfatahmadwani@gmail.com

V. H. Badshah

School of Studies in Mathematics,

Vikram University Ujjain,

Ujjain, India

email:vhbadshah@gmail.com

S. Halici

Department of Mathematics, Faculty of Arts and Sciences,

Sakarya University,

54187 Sakarya, Turkey

email:shalici@pau.edu.tr

Paula Catarino

Department of Mathematics, School of Science and Technology,

University of Trás-os-Montes e Alto Douro (Vila Real Portugal)

email: pcatarino23@gmail.com 